\title{
Allergy to extensively hydrolyzed formula in an infant with cow's milk allergy and transient hypogammaglobulinemia
}

\author{
Mona Akad ${ }^{1}$, Paul Alexandru Simion ${ }^{1}$, Fawzy Akad², Monica Alexoae ${ }^{*}, 1,3$ \\ 1"Grigore T. Popa" University of Medicine and Pharmacy, Iași, ${ }^{2}$ General Surgery Department of CFR \\ Hospital, lasi, ${ }^{3} I^{\text {nd }}$ Immunology-Allergology Department, "Sf. Maria" Emergency Hospital for Children, \\ lasi, Romania.
}

\begin{abstract}
Atopic dermatitis (AD) is a chronic inflammatory skin disorder with an increased prevalence among infants, being the first manifestation of the „allergic march”. Food allergies are the main triggers for this pathology and it is commonly known that the younger the infant, the higher the risk of developing $A D$. Treatment of $A D$ as a consequence of allergy to cow's milk protein (CMPA) consists in administrating a therapeutic formula such as an extensively hydrolyzed formula (eHF), or an Amino Acid Formula (AAF), hygienic measures, and antiinflammatory treatment. We present the case of a 2 months old infant, artificially fed since birth, submitted to the Second Clinic of Pediatrics, "Sf. Maria" Emergency Hospital for Children lasi, for a moderate form of AD due CMPA, associated with transient hypogammaglobulinemia (TH). The diagnosis was established using Hanifin and Rajka criteria, the dosage of specific serum $\operatorname{lgE}(\mathrm{s} \operatorname{lgE})$ for cow's milk proteins (CMP), and the positive response to diet replacement. The severe clinical manifestations were caused by the cutaneous infection with Staphylococcus aureus, and exacerbated by immunodeficiency. Under antibiotic treatment and the introduction of eHF in the infant's diet, we record a positive evolution. The infant is readmitted in the Pediatrics Clinic 72 hours after discharge, with moderate dehydration associated with diarrheic stools, requiring the replacement of eHF with an AAF. Although rarely encountered, allergies to eHF are reported in $10 \%$ of patients with $A D$, and usually manifest with gastrointestinal symptoms. AAF is an efficient therapeutic replacement for this category of infants.
\end{abstract}

Keywords: immunodeficiency, cow's milk proteins, atopic dermatitis, food allergy

\section{Introduction}

Atopic dermatitis is a chronic inflammatory skin disorder that usually affects children, but can occur at all ages [1]. Most of the time AD is the primary manifestation in patients that suffer from food allergies and that later on develop other conditions such as allergic rhinitis or asthma, composing the "allergic march" [2].

Received: October 2015; Accepted after review: December 2015; Published: December 2015

${ }^{*}$ Corresponding author: Monica Alexoae, MD, PhD, II $^{\text {nd }}$ Immunology - Allergology Department, "Sf. Maria" Emergency Hospital for Children, lasi, Romania; "Grigore T. Popa" University of Medicine and Pharmacy, 16 University Street, 700115, Iași, Romania. Phone: 0723162804

Email: m alexoae@yahoo.com
The major hallmarks of this atopic disease are xerosis, pruritus and chronic relapsing eczematous lesions of the teguments. Cutaneous lesions have the ability to show different clinical manifestations depending on the child's age. In infants and young children the eruption consists in papular-erythematous lesions that are usually located on the facial area and convex areas such as the forehead, scalp and malar region and in older children the lesions have a chronic character and are disposed on concave areas (elbows, popliteal and retroauricular area) [3].

Food allergies (FA) are the most frequent triggers for $A D$ at young ages. The implication of food triggers is viewed differently throughout the world but one thing is clear: the 
younger the child, the higher the amplitude of the allergic event [4]. According to the metaanalysis as part of the EuroPrevall program the prevalence of $\mathrm{FA}$ is $3 \%$ to $6 \%$, in infants being greater than in adults. At the age of one year, the main allergenic triggers are cow's milk proteins and Hen's egg protein [5].

Cow's milk is usually the infant's first meal, and it contains more than 30 proteins with high allergenic potential, beta-lactoglobulin being the major allergenic trigger. The similarity of certain protein fraction structures is an explanation towards the cross allergies between cow's milk and the milk of other mammalian species (goat, sheep) and of course between cow's meat and soya [6].

$A D$ is an $\lg E$ mediated skin disorder with a deviated immune response that consists in increased systemic Th2 cells and a combination of Th2 and Th1 cells in skin lesions [7]. Flow cytometry studies reveal that patients with $A D$ in basal circumstances have an increased level of Th2, compared to the control groups without AD. The complex mixture of cytokines secreted by these cells including IL-4, IL-5 and IL-13 explain two clinical findings in $A D$ patients: hypereosinophilia (linked to the hyperproduction of IL-5) and hypergammaglobulinemia $E$ (linked to the IL-4) $[7,8]$. If in acute lesions there is an increased level of Th2 cells, in chronic lesions there is a predomination of IFN y secreting Th1 cells [2].

Another inadequate immune response that can be found in $A D$ patients is hypogammaglobulinemia. Studies reveal that hypogammaglobulinemia is more frequent in patients with $A D$ compared with the control groups, but it is not correlated with the severity of the skin disorder [9]. TH is typically found by the age of 6 months, and most of the patients outgrow this condition by the age of two years. The diagnosis of $\mathrm{TH}$ is made a posteriori, when the immunoglobulin levels normalize [10]. This condition, along with the underlying dysfunctional immune response of the patient makes the skin lesions susceptive to Staphylococcus aureus infection [11].

The diagnosis of $A D$ is mainly suggested by the clinical presentation and status of the patient. For the clinical diagnosis the guidelines recommend the use of Hanifin and
Rajka criteria which requires 3 out of 4 major criteria and 3 out of 23 minor criteria to be met, and the SCORAD index for the assessment of the severity of $A D$ [12].

Discovering the allergen is mandatory for a successful treatment and it involves a detailed anamnesis targeting the diet, a thorough clinical examination and specific laboratory investigations [12]. According to the guidelines for the diagnosis and management of the food allergy in the United States 2010, for the identification of foods that may induce Ig E-mediated allergenic reactions, only the skin prick test (SPT) and the determination of slgE from blood samples are recommended. Other common immunological investigations such as intradermal test, that has a high chance of adverse effects, or patch test, that has a high variance in sensitivity and specificity, are not recommended for clinical practice. No literature supports the usage of SPT, slgE dosage and patch test in combination, and they have no advantage over the usage of slgE or SPT alone [13, 14].

The diagnosis needs to be confirmed or infirmed by food-challenge procedures. These tests can be open, single or double-blinded but the double-blind placebo-controlled food challenges are the "gold standard" in food allergy diagnosis, and can only be performed in an allergology department by a specialized medical team that can prevent any possible life threatening reactions. These tests can be omitted if the patient has a history of anaphylaxis or if there are very strong arguments for the CMPA diagnosis [14].

The treatment of $A D$ includes avoidance of the allergen, the control of xerosis by using moisturizers, emollients, bathing therapies, anti-inflammatory medication such as topical corticotherapy or topical calcineurin inhibitors, and in case of a bacterial infection, antibiotic therapy. Usually, the severity of the illness dictates aggressive treatment $[2,12]$.

\section{Case report}

A two months old infant from rural area is submitted to "Sf. Maria"-Hospital - Second Clinic of Pediatrics with an itching papular erythematous eruption of the face, cervical 
and the inguinal region with well-defined margins, which did not relent to local corticoid treatment with hydrocortisone butyrate. Clinical aspect is very suggestive for atopic dermatitis.

Family history: mother of 20 years old and father of 40 years old deny any history of atopic syndromes in the family and they are both apparently in good health and without any other family pathology that might be conclusive for our case.

Personal history: couple's first child, from an unmonitored pregnancy, naturally delivered at 36 weeks gestational age (there was no need of incubation), cranial presentation, birth weight of $2950 \mathrm{~g}$, height of $50 \mathrm{~cm}$ and an APGAR score of 8 (apparently without prenatal pathology). The infant was artificially fed from birth with basic formula, and fulfilled the vaccination scheme until present. The infant has no pathologic personal history.

Disease's history. The insidious debut of the affection that started at the age of one month and three weeks was marked by the appearance of an itching papularerythematous eruption of the face, cervical area, and inguinal region, with well-defined margins and with a homogenous aspect. General practitioner recommends topical applications with hydrocortisone butyrate, but the lesions continued to aggravate and extend to the perineal region, for which the infant was submitted to "Sf. Maria" Hospital.

Admission status. We had to examine a female infant with an altered general status, afebrile, with normal anthropometric parameters according to her age (weight of $5000 \mathrm{~g}$, height of $58 \mathrm{~cm}$, and weight gain index of 1). During the clinical examination we encountered a generalized cutaneous xerosis associated with intense itching papular erythematous eruption of the teguments in the facial and cervical area (Figure 1), the trunk, more pronounced in the flexion folds (inguinal region) (Figure 2 ), extension surfaces, and a cradle cap (retention of hyperkeratosis) on the vertex of the scalp (Figure 3).

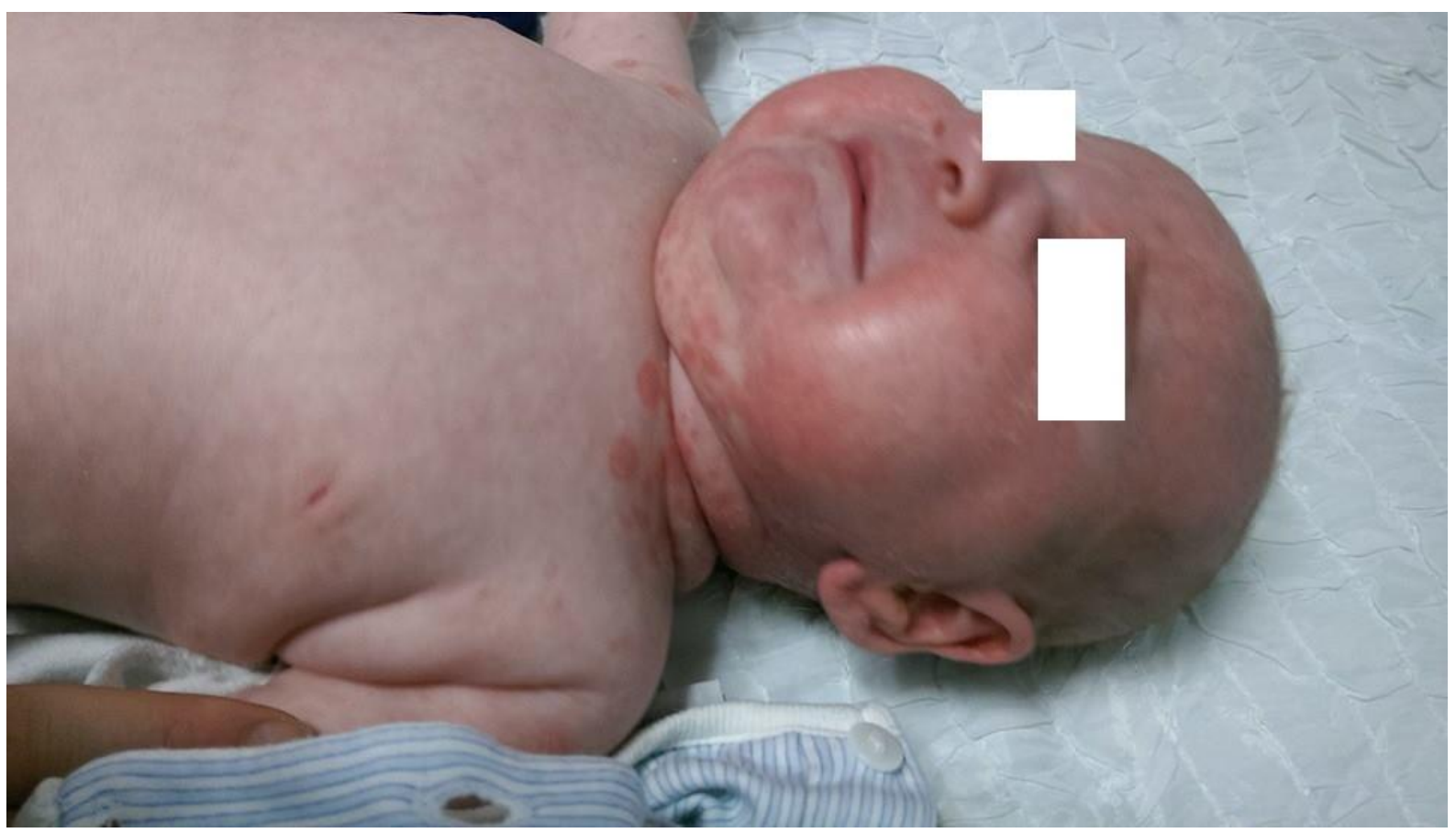

Fig. 1. Objective examination reveals an papular erythematous eruption in the facial and cervical area. 


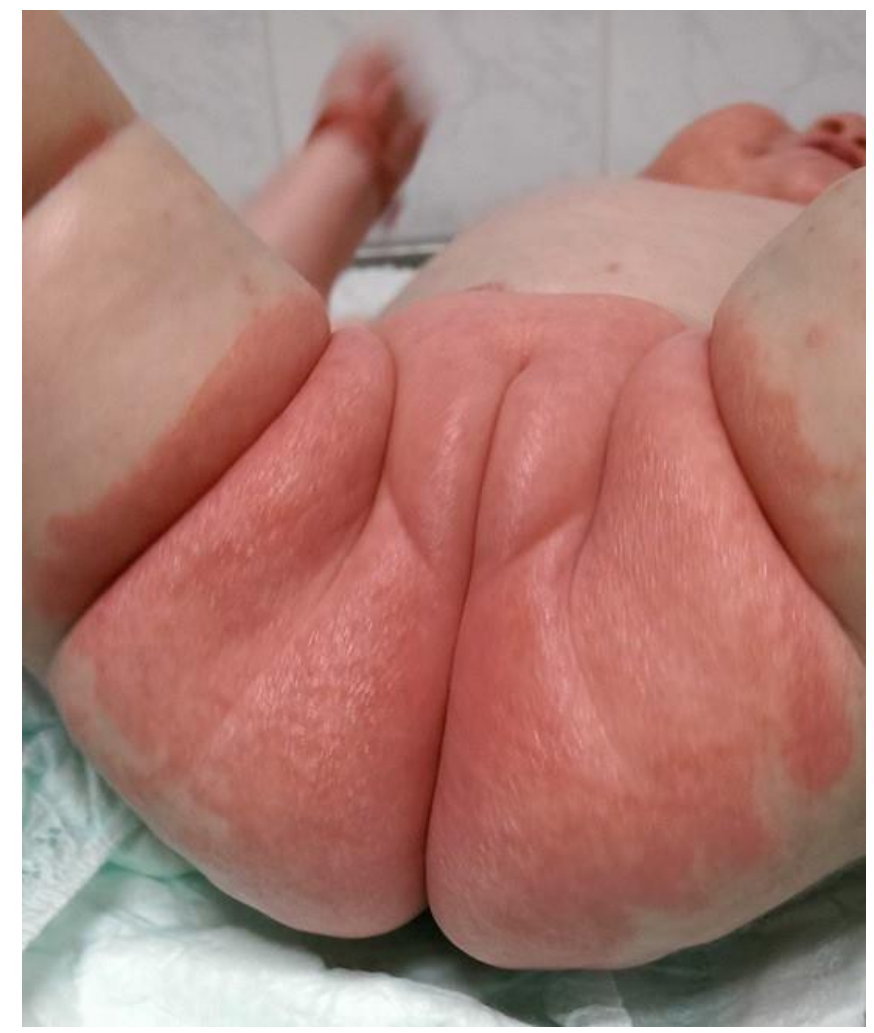

Fig. 2. Objective examination reveals an intense homogenous papular erythematous lesion with well-defined margins in the genital-inguinal area with inferior extension to the perineal area, highly suggestive for a cutaneous infection.

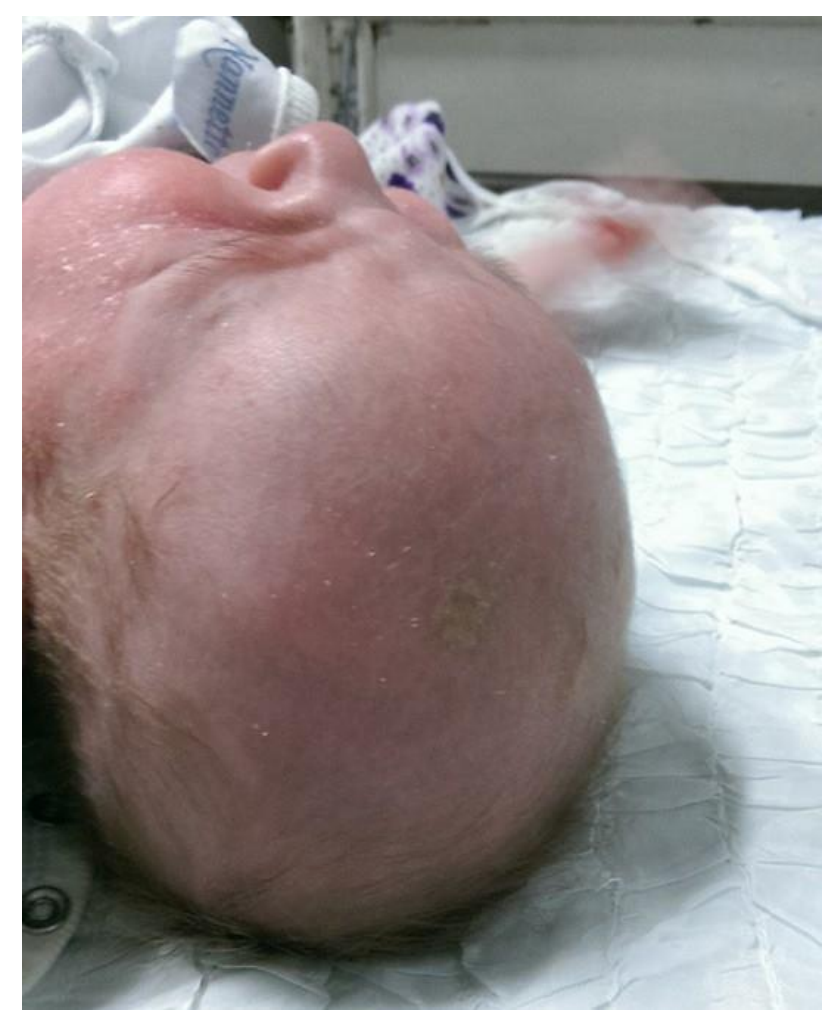

Fig. 3. Objective examination reveals hyperkeratosis of the head vertex. 
Considering the anamnesis (the infant was artificially fed with basic formula since birth) and the clinical examination of the infant we release the presumptive diagnosis of Atopic Dermatitis complicated with cutaneous infection. Therefore we request specific laboratory tests and we discover a series of pathological values. Hematology tests reveal a moderate nonspecific inflammatory syndrome $(\mathrm{VSH}=25 \mathrm{~mm} / 1 \mathrm{~h}$, and $\mathrm{CRP}=2 \mathrm{mg} / \mathrm{dl})$, and high levels of hepatic enzymes (Table 1). Immunological investigations reveal hypogammaglobulinemia with $\lg \mathrm{A}$ and $\lg G$ deficit (Table 2).

We also withdraw a sample from the cutaneous lesions and the results turns out positive for Staphylococcus aureus, sensitive to Clindamycine.
Considering the high levels of hepatic enzymes we also request an abdominal ultrasound that shows no pathological modifications.

By using the Hanfin and Rajka diagnosis criteria we managed to find three major criteria: pruritus, the specific distribution of the eczematous lesions and chronic symptoms, and four minor criteria: xerosis, early debut, high levels of slgE and food intolerance that helped us decide for the AD diagnosis.

Positive diagnosis. Having reviewed the anamnesis, clinical examination and the laboratory test results we can confirm the final diagnosis of Atopic dermatitis with Staphylococcus aureus skin infection, Allergy to cow's milk proteins, and transient hypogammaglobulinemia.

Table 1. High levels of TGO, TGP and GGT which suggest a hepatic cytolysis syndrome.

\begin{tabular}{llll}
\hline Test & Results & Measure Unit & Normal Values \\
\hline TGP & 113 & $\mathrm{U} / \mathrm{l}$ & $5-38$ \\
TGO & 69 & $\mathrm{U} / \mathrm{l}$ & $5-35$ \\
ALK & 603 & $\mathrm{U} / \mathrm{L}$ & $40-600$ \\
GGT & 55 & $\mathrm{U} / \mathrm{L}$ & $7-38$ \\
\hline
\end{tabular}

Table 2. High levels of total IgE and slgE against beta-lactaglobulines and alfa-lactalbumines confirm the diagnosis of allergy to cow's milk proteins. Low levels of IgA and IgG suggest a hypogammaglobulinemia.

\begin{tabular}{llll}
\hline Test & Results & Measure Unit & Normal Values \\
\hline Gamma globulines & $10.5 \%$ & $\%$ & $12-22 \%$ \\
Ig G & 600 & $\mathrm{Ul} / \mathrm{ml}$ & $800-1800$ \\
Ig A & 48 & $\mathrm{UI} / \mathrm{ml}$ & $70-400$ \\
Ig E & 100 & $\mathrm{UI} / \mathrm{ml}$ & $<60$ \\
IgE-Beta-lactaglobulin & 0.86 & $\mathrm{kUA} / \mathrm{l}$ & $<0.35$ \\
Ig E-Alfa-lactalbumin & 0.50 & $\mathrm{kUA} / \mathrm{l}$ & $<0.35$ \\
\hline
\end{tabular}

\section{Treatment}

A specific diet that will accomplish the allergen avoidance was implemented by replacing the old basic formula with an eHF (3 measures for each meal, 7 meals of $120 \mathrm{~mL}$ per day).

For the prevention and control of xerosis, hygienic and pharmacological measures were implemented. The parents were advised to use wet sterile gauze at room temperature in order to brush gently the dry skin of the infant 3 to 4 times a day, to continuously apply, even in quiet periods, emollient creams which contain polidocanol, urea and salicylic acid, especially in hyperkeratosic lesions, and to avoid dermatocosmetics which contain parabens or preservatives. The clothing which makes direct contact with the skin should be made out of cotton, and must be washed with hypoallergenic detergent. For pruritus control, the overheating of the room should be prevented and the bathing water should be maintained at 33 to $35^{\circ}$ Celsius.

Antiinflamatory treatment was performed with topical corticotherapy, one to three 
applications per day, and oral Dexamethasone $0.2 \mathrm{mg}$ per $\mathrm{kg}$, twice a day, for 5 days. The antiinflamatory treatment was established after calculating the SCORAD index which allowed us to categorize the severity of $A D$. In our case, the patient had a score of 19 , suggestive of moderate $A D$, for which the guidelines recommend a short-term oral corticoid therapy.

The cutaneous infection was treated with targeted antibiotic therapy using Clindamycine $10 \mathrm{mg}$ per $\mathrm{kg} /$ day twice a day, intravenous, for 7 days.

The hypogammaglobulinemia doesn't require any treatment, targeted antibiotic therapy being sufficient for controlling acute infections.

\section{Evolution and prognosis}

Evolution during the admission was favorable, with the amelioration of the skin lesions. The infant was discharged afebrile, with good digestive tolerance to the eHF and with an ascending curve of the ponderal index. At 72 hours, she is readmitted with mucous diarrhea (6-7 stools/day), moderate dehydration, and reappearance of the skin lesions. The absence of viral or bacterial cause for the diarrheic syndrome, and the findings of intestinal microhemorrhages, determined that the condition is immune mediated, so the eHF was changed to an AAF, followed by an amelioration of the symptoms.

The long term evolution depends on respecting the new diet based on AAF and the comprehension degree the parents will show towards the new lifestyle that needs to be insured for the infant. The amelioration or exacerbation of the atopic status of the infant depends on several factors such as: exposure to different allergenic substances, bacterial or viral infections, high temperatures and an inappropriate hygiene, new vaccines, clothes made out of synthetic materials or exposure to emotional and physical stress.

\section{Discussions}

In the above presented case, other probable diseases were eliminated because the symptoms and clinical signs didn't fit the infant's pathological profile.

Seborrheic dermatitis (SD) was eliminated due to the fact that in SD the cradle cap is greasy and yellow, and in our case it was dry and crusted, specific to AD. Contact dermatitis usually appears in certain areas that were in contact with potentially allergenic materials such as nickel snaps in undershirts, but it avoids xerosis and facial involvement. In our case the papular erythematous eruption was involving the cervical area of the head and the face. Leiner disease symptoms include SD of the scalp, systemic infections, central nervous system deficiencies, severe diarrhea, peeling of the skin accompanied by itching, and corneal ulcers. In our case the infant had no central nervous system deficiencies, and the lesions were dry and without the tendency to a quick extension. Reiter Disease tegument manifestations similar to atopic dermatitis: dry, erythematous skin lesions that can occur in small patches but most of the time cover large areas and it is accompanied by bullous lesions. It is commonly encountered in the toes, nails, and soles of feet, unlike our case. Letterer-Siwe Disease is an association of cutaneous, systemic and bone lesions. It is associated with tegumentary manifestations such as erythematous papular eruptions involving mostly the trunk, but in the same time it is associated with significant anemia, neutropenia, hepatosplenomegaly and pulmonary manifestations, which is not our case. Wiskott Aldrich syndrome is eliminated by the absence of thrombocytopenia, and hemorrhagic syndrome such as spontaneous nose bleeding or bloody diarrhea [16].

The early debut (only two months old), severe symptoms, female sex and the high levels of $\lg E$ are predictive factors for the persistency of this pathology later on [17]. Almost $1 / 3$ of the infants diagnosed with $A D$ at the age of less than 1 year will develop asthma or allergic rhinitis in childhood.

$A D$ is a common chronic relapsing inflammatory skin disorder mainly caused by food allergies. The prevalence of $A D$ has increased over the past three decades in industrialized countries [18]. The disease manifests at a young age, $45 \%$ of the patients developing symptoms in the first 6 months, 
$60 \%$ in the first year and $85 \%$ before 5 years. Almost $70 \%$ of the patients have a spontaneous remission of the condition before adolescence, and in rare cases $A D$ can start manifesting in adulthood as late-onset atopic dermatitis [19].

$A D$ is an $\lg E$ mediated disease with a complex immunopathogenesis. There is a combination of genetic, immune and environmental factors which lead to the dysregulation of the immune system and the disruption of the skin barrier mechanisms.

The genetic basis of $A D$ is sustained by the fact that the concordance rate of this condition is $70 \%$ between monozygotic twins and $15 \%$ between dizygotic twins [20]. Four major genomic studies provide genome-wide significant evidence of linkage with chromosomes 1q, 3q, 3p and 17q. Most of these genes correspond with loci for psoriasis, but these diseases are rarely related. Recent studies revealed that several genes located on CRS 5q31-33 are related wih AD. These genes encode cytokines produced by Th2 cells: IL-4, IL-5 and IL-13, usually found in acute lesions [21]. Flowcytometry studies back this hypothesis up by demonstrating the higher concentration of type 2 cytokinic profile in the skin of patients with $A D$ in basal circumstances compared to control groups without $A D$ [8].

Another known gene well correlated with the appearance of $A D$ is the fillagrin gene, encoded on 1q21.3 chromosome. This is a major predisposition factor for early-onset $A D$, and it also a cause of Ichthyosis vulgaris, a disease that manifests with itching, dryness and scaling of the skin [22].

Skin barrier alteration is another predisposition factor for AD. Deficiencies in ceramides and cathelicidin in the skin leads to transepidermal water loss which can increase the penetration of allergens and microbes. This barrier defect explains why $80 \%$ of $A D$ skin lesions become infected with Staphylococcus aureus [23].

Another deviated immune response correlated with $A D$ is $T H$. This is an immunodeficiency disorder characterized by a decreased serum $\lg G$ with or without a decrease of $\lg A$ and $\lg M$. TH usually affects infants with the age between 3 and 6 months, and tends to resolve spontaneously by the age of 2 years. For that reason the diagnosis of "transient" can be put only retrospectively.

Hypogammaglobulinemia can be primary, secondary, transient, and physiological (due to rapid catabolization of the transplacental acquired $\lg G$ ) [24]. The most frequent type is $\mathrm{TH}$ and is strongly associated with atopic syndrome, risk of infections and autoimmune disorders [10, 11].

In our case, the patient presented hypogammaglobulinemia associated with CMPA and Staphylococcus aureus infection of the skin lesions. The guidelines recommend the use of targeted antibiotherapy without the replacement therapy of intravenous gammaglobulines. The replacement therapy is only needed when the patient develops severe life-threatening or severe recurrent infections despite the targeted antibiotic therapy.

The clinical diagnosis of atopic dermatitis was set according to CMPA by using the Hanfin and Rajka criteria: itching, specific distribution of the eruption, chronic symptoms, xerosis, early debut, high level of $\lg \mathrm{E}$ and food intolerance. Since their first description in 1980, Hanifin and Rajka remained the most recognized diagnostic test. The major inconvenient is the large number of criteria, which makes it difficult to be widely used. Although they have a sensitivity of $96 \%$ and specificity of $93.75 \%$ with a statistical advantage over the UK working party's diagnostic criteria [12, 13, 25], it cannot be solely used for the diagnosis of $A D$, immunological investigations and food challenge procedures being mandatory in order to establish a positive diagnosis.

For the assessment of the severity of $A D$ the most commonly used scales are Eczema Area and Severity Index (EASI), Six Area, Six Sign Atopic Dermatitis (SASSAD) severity score, and SCORAD index. The guidelines suggest the SCORAD index for clinical practice, the rest being used for trials and epidemiologic studies [12].

Because the most common cause of $A D$ is food allergy, the trigger must be found. Methods of identifying the allergens are: skin prick test, intradermal tests, dosage of total and specific Ig E, atopy patch test, and food elimination diets. Out of these explorations 
only the usage of skin prick test and dosage of specific Ig $E$ in blood samples are recommended, because they have the least chance of adverse reactions and they show higher specificity and sensitivities over the others. The use of these tests in combination is not advised because they are inconvenient for the patient, time-consuming, and they have a slightly higher rate in specificity and sensitivity. In our case we investigated the specific $\lg E$ for beta lactoglobulines and alpha lactobulines, for which we found high values, highly suggestive for CMPA.

For a positive diagnosis, oral challenge procedures are needed. The double-blind placebo controlled food challenge procedure is the gold standard test for food allergy, but it requires an allergology department with a specialized medical team in order to prevent and control any adverse reactions such as anaphylaxis. The test can be omitted only if there are strong arguments for the atopic disease or if there is a history of anaphylaxis. In our case, the patient had signs and symptoms specific for $A D$, and correlating the Hanifin and Rajka criteria with an increased level of specific $\lg \mathrm{E}$ to CMP and amelioration of the symptoms with the removal of the cow's milk from the diet, the positive diagnosis was made without the necessity of an oral challenge procedure $[12,14,26]$.

The treatment of $A D$ due to CMPA consists in pharmacologic and nonpharmacologic interventions. Nonpharmacologic measures include diet changing and hygienic measures.

The first step in management of CMPA patients is to change the formula used with a therapeutic one. Guidelines define therapeutic formula as one tolerated by at least $90 \%$ of patients with CMPA with a confidence of $95 \%$ but only a few eHF with casein and AAF can meet can fit the standards.

The use of therapeutic eHF is recommended as the first choice in the diet plan because it's cheaper, making the parents compliant to the diet, and only $10 \%$ of the patients with CMPA present reactions to it [27]. AAF is required only if the lesions are severe, persist or aggravate, if the cost benefit-ratio favors $A A F$, and if the patient doesn't tolerate the eHF. In our case the patient was readmitted with diarrhea and gastrointestinal symptoms, as an adverse reaction to the eHF, which raised the necessity of an AAF diet. Studies reveal that residual allergens in eHF are most likely to cause gastrointestinal manifestations [28, 29], symptomatology consistent with our case.

Another mandatory step in the therapy is the prevention and control of xerosis. These measures include moisturizers, emollients with glycerol, which lubricate and soften the skin, occlusive agents like petrolatum, which form a layer on the skin in order to slow down the evaporation of water, and humectants, containing urea or lactic acid, which attracts and hold water. Applying these creams reduce the inflammation in the skin and the required intake of corticoid treatment.

Another good measure to prevent xerosis is frequent short bathing, 5 to 10 minutes, with water at 33 to $35^{\circ}$ Celsius. At 20 minutes after bathing it is recommended to apply local corticoid lotions, or emollients without toweling-dry the skin, because it enhances the antiinflamatory effect. This technique is known as "soak and smear" and its use is highly recommended [30].

Pharmacologic treatment includes corticotherapy, calcineurin inhibitors, antihistamines and modern therapies (currently in trials).

Corticotherapy is the main, standard treatment in $A D$, and it can be administered topically, orally or intravenous, depending on the severity of the disease. Usually only topical corticoid treatment is required, the others being used for moderate to severe forms. For lotions, there is no standard for quantity of application. Guidelines suggest the usage of one fingertip unit, which corresponds to $0.5 \mathrm{~g}$ of substance, which should be applied to a surface area corresponding to two adult palms. The corticoid agent used should be the least potent one that is effective, in order to reduce adverse reactions.

Adverse reactions appear in long-term use of corticotherapy and include: Cushing syndrome, greater risk of infections, increased scarring time and reduction of skin lesion healing, ecchymosis, subcutaneous tissue atrophy, glucose intolerance, insomnia [31]. 
Calcineurin inhibitors are the second-line therapy recommended when there are adverse effects to corticoid treatment, or when a continuous long term corticoid therapy was used. Tacrolimus and pimecrolimus are representative for the topical calcineurin inhibitors, and they have benefic effects compared to the standard therapy. Tacrolimus has a higher potency, and it is recommended in moderate to severe forms of $A D$, and pimecrolimus in mild to moderate cases. The main adverse effect is the burning sensation, which may determine the parents to disrupt the treatment, which will result in the reoccurrence of the skin lesions $[2,12]$. Other anti-inflammatory agents are antihistamines, which show little effect and are not recommended.

Currently in trials are certain "modern" therapies like: immunotherapy, anti $\lg \mathrm{E}$ monoclonal antibody therapy, modified food protein vaccines, plasmid DNA immunization, peptide immunotherapy and trichuris suis ova, but they require much more testing in order to achieve clinical practice.

In our case, we recommended topical corticotherapy, one to three applications per day, applied in thin uniform layers until the disappearance of the skin lesions. Because it was a moderate form of $A D$, a short-term oral dexamethasone therapy was needed. The evolution under AAF diet was favorable and the patient didn't manifest any adverse reactions to treatment.

The prognosis and long-term evolution of $A D$ is favorable, $60 \%$ of these patients tend to outgrow this condition into adulthood. There are some prognostic factors strongly related with the persistence of AD: early age of onset, family history of $A D$, female sex, widespread

\section{References}

1. Schultz-Larsen F, Hanifin J. Epidemiology of atopic dermatitis. Immunol Allergy Clin North Am 2002; 22:1-24.

2. Schneider L, Tiles S, Lio $P$, et al. Atopic Dermatitis: A practice parameter update 2012. J Allergy Clin Immunol 2013; 131:296-299.

3. Akdia CA et al. Diagnosis and treatment of atopic dermatitis in children and adults: European Academy of Allergology and Clinical dermatitis, persistence of dry itching skin in adult life and associated asthma or allergic rhinitis. As an addition to these factors, if the dermatitis is due to CMPA or Hen's egg proteins it will have a worse outcome in terms of persistence and severity, compared to other causative allergens.

Our patient has three out of six of these prognostic factors, and an association with CMPA which suggests a moderate risk for the prolongation of the disease, which will require a more tight supervision.

\section{Conclusion}

Atopic dermatitis due to CMPA is common in infants and rarely persists in adulthood. Although guidelines recommend the diet with eHF, in $10 \%$ of the cases gastrointestinal manifestations appear as a reaction to it. In our case, the patient was readmitted 72 hours after discharge with diarrhea and moderate dehydration, for which the introduction of AAF was necessary. Associated with the atopic disease, the infant presented an infection of the skin lesions with Staphylococcus aureus. This is the most frequent complication of AD, and in our case it was favored by the hypogammaglobulinemia. Often $\mathrm{TH}$ is associated with atopic diseases, due to the underlying dysfunction of the immune system, and these types of patients should be strictly supervised because it brings a higher risk of infection which in some cases can be lifethreatening. The appearance of $A D$ at a young age raises the possibility of being the first manifestation of the "allergic march", and is one of the main risk factors for its progression to adulthood.
Immunology/American Academy of Allergy, Asthma and Immunology/PRACTALL Consensus Report. Allergy 2006; 61:969-987.

4. Fleischer DM, Bock SA, Spears GC, Wilson CG, Miyazawa NK, Gleason MC, et al. Oral food challenges in children with a diagnosis of food allergy. J Pediatr 2011; 158:578-583.

5. Sicherer SH. Epidemiology of food allergy. $J$ Allergy Clin Immunol 2011; 127:594-602. 
6. Eichenfield LF, Hanifin JM, Luger TA, Stevens $\mathrm{SR}$, Pride HB. Consensus conference on pediatric atopic dermatitis. J Am Acad Dermatol 2003; 49:1088-95.

7. Novak $\mathrm{N}$, Bieber $\mathrm{T}$, Leung DY. Immune mechanisms leading to atopic dermatitis. $J$ Allergy Clin Immunol 2003; 112(6):S128-39.

8. Schuerwegh AJ, De Clerck LS, De Schutter L, Bridts $\mathrm{CH}$, Verbruggen A, Stevens WJ. Flow cytometric detection of type 1 (IL-2, IFNgamma) and type 2 (IL-4, IL-5) cytokines in Thelper and T-suppressor/cytotoxic cells in rheumatoid arthritis, allergic asthma and atopic dermatitis. Cytokine 1999; 11(10):783-8.

9. Celiksoy MH, Topal E, Sancak R, Catal F, Sogut A. Relationship between hypogammaglobulinemia and severity of atopic dermatitis. Ann Allergy Asthma Immunol 2014; 113(4):467-9.

10. Wang AS, Liang MG, Schneider LC. Severe atopic dermatitis and transient hypogammaglobulinemia in children. Pediatr Dermatol 2012; 29(1):73-8.

11. Gong JQ, Lin L, Lin T, et al. Skin colonization by Staphylococcus aureus in patients with eczema and atopic dermatitis and relevant combined topical therapy: a double-blind multicentre randomized controlled trial. $\mathrm{Br} \mathrm{J}$ Dermatol 2006; 155(4):680-7.

12. Lawrence F, Wynnis L, Timothy G, et al. Guidelines of care for the management of atopic dermatitis. J Am Acad Dermatol 2014; 70:338-51.

13. Hanifin JM, Rajka G. Diagnostic features of atopic dermatitis. Acta Derm Venereol 1980; 92:44-7.

14. Boyce JA, Assa'ad A, Burks AW, et al. Guidelines for the diagnosis and management of food allergy in the United States: report of the NIAID sponsored expert panel. J Allergy Clin Immunol 2010; 126:S1-58.

15. Bidat E, Rance' F, Barane's T, Goulamhoussen S. Goat's milk and sheep's milk allergies in children in the absence of cow's milk allergy. Rev Fr Allergol Immunol Clin 2003; 43:273-277.

16. Alfons $K$, Bernice $K$. The differential diagnosis of atopic dermatitis in childhood. Dermatologic Therapy 2006; 19: 73-82.

17. Lam J, Freidlander S. Atopic dermatitis: A review of recent advances in the field. Pediatr Health. 2008; 2(6):733-747

18. Larsen FS, Holm NV, Henningsen K. Atopic dermatitis: a genetic epidemiologic study in a population-based twin sample. J Am Acad Dermatol 1986; 15:487-94.
19. Bieber T. Mechanisms of disease: atopic dermatitis. N Engl J Med 2008; 358:1483-1494.

20. Schultz Larsen FV, Holm NV. Atopic dermatitis in a population based twin series: concordance rates and heritability estimation. Acta Derm Venereol Suppl 1985; 114:159-159.

21. Morar N, Willis-Owen SA, Moffatt MF. Cookson WO. The genetics of atopic dermatitis. J Allergy Clin Immunol 2006; 118:24-34.

22. Sandilands A, Terron-Kwiatkowski A, Hull PR, et al. Comprehensive analysis of the gene encoding filaggrin uncovers prevalent and rare mutations in ichthyosis vulgaris and atopic eczema. Nat Genet 2007; 39(5):650-4.

23. Gong JQ, Lin L, Lin $T$, et al. Skin colonization by Staphylococcus aureus in patients with eczema and atopic dermatitis and relevant combined topical therapy: a double-blind multicentre randomized controlled trial. $\mathrm{Br} \mathrm{J}$ Dermatol 2006; 155(4):680-7.

24. Simonte SJ, Cunningham-Rundles C. Update on primary immunodeficiency: defects of lymphocytes. Clin Immunol 2003; 109(2):10918.

25. Kanwar AJ, Handa S, De D. Comparative efficacy of Hanifin and Rajka's criteria and the UK working party's diagnostic criteria in diagnosis of atopic dermatitis in a hospital setting in North India. J Eur Acad Dermatol Venereol 2006; 20(7):853-9.

26. Hill DJ, Hosking CS, de Benedictis FM, et al. EPAAC Study Group. Confirmation of the association between high levels of immunoglobulin $E$ food sensitization and eczema in infancy: an international study. Clin Exp Allergy 2008; 38:161-168.

27. Boissieu D, Dupont C. Allergy to extensively hydrolysed cows' milk proteins in infants: safety and duration of amino acid-based formula. $J$ Pediatr 2002; 141:271-3.

28. Vanderhoof JA, Murray ND, Kaufman SS, et al.Intolerance to protein hydrolysate infant formulas: an underrecognized cause of gastrointestinal symptoms in infants. $J$ Pediatr 1997; $131(5): 741-4$.

29. Niggemann B, von Berg A, Bollrath $C$, et al. Safety and efficacy of a new extensively hydrolyzed formula for infants with cow's milk protein allergy. Pediatr Allergy Immunol 2008; 19(4):348-354.

30. Gutman AB, Kligman AM, Sciacca J, James WD. Soak and smear: a standard technique revisited. Arch Dermatol 2005; 141(12):1556-9.

31. Matusiewicz R, Stempniak M, Lebiedowski K, Czajkowski $M$. The most frequent complications during long-term corticotherapy. Wiad Lek 1989; 42(5):273-7. 